\title{
Perfil Socioeconômico e Expectativa de Carreira dos Estudantes de Medicina da Universidade Federal da Bahia
}

\author{
Socioeconomic Profile and Career Expectations of Medical \\ Students of the Universidade Federal da Bahia
}

\author{
Renata Meira Veras ${ }^{I}$ \\ Clara Couto Fernandez $z^{I}$ \\ Caio Cezar Moura Feitosa ${ }^{I}(\mathbb{D}$ \\ Sheyla Fernandes ${ }^{I I}$ (iD
}

\section{PALAVRAS-CHAVE}

- Educação Médica.

- SUS.

- Estudantes.

- Universidade.
${ }^{1}$ Universidade Federal da Bahia, Salvador, Bahia, Brasil.

${ }^{\text {II }}$ Universidade Federal de Alagoas, Maceió, Alagoas, Brasil. 


\section{KEYWORDS}

- Medical Education.

- SUS.

- Students.

- University.

Recebido em 21/11/19

Aceito em 21/1/20

\section{INTRODUÇÃO}

O campo de estudos em educação médica no Brasil tem se constituído como um terreno árido pelos questionamentos e conflitos suscitados pelo debate acerca da formação do "bom médico". Esse debate instaura-se principalmente com as experiências advindas da criação do Sistema Único de Saúde (SUS) ${ }^{1}$. No Brasil, a implementação do SUS veio acompanhada da reflexão sobre a formação profissional. Como garantido constitucionalmente, o SUS se apresenta como ordenador de recursos humanos na área da saúde ${ }^{2,3}$. Assim, quando se reconhece essa função, faz-se necessária a reestruturação curricular no curso de Medicina. Essa reestruturação envolve uma série de decisões que perpassam o poder público, os gestores dos serviços, a comunidade e, especialmente, as instituições formadoras ${ }^{1}$.

Nesse sentido, vários esforços têm sido desenvolvidos para repensar os modelos pedagógicos na formação desses profissionais, objetivando maior articulação da formação superior com o sistema de saúde ${ }^{1,4}$. Essa articulação está presente nas orientações das Diretrizes Curriculares Nacionais para o curso de Medicina (DCN) publicadas em 2001 e atualizadas em 2014. Impulsionados pela publicação das diretrizes, os currículos das universidades passaram por reestruturações, posto que é notória a ênfase dada à necessidade de atrelar o currículo médico às reais necessidades de saúde da população ${ }^{5,6}$.

Hoje, no Brasil, a graduação em Medicina tem duração de seis anos, dos quais os quatro primeiros correspondem ao ciclo básico, e os dois últimos, ao internato. A carga horária mínima é de 7.200 horas, podendo chegar a nove mil horas em algumas instituições?

A Faculdade de Medicina da Bahia (Fameb) é um exemplo de instituição que modificou a sua estrutura curricular. Criada em $1808 \mathrm{com}$ a vinda da família real portuguesa para o Brasil, a estrutura curricular dessa instituição passou por várias reformas. A última delas ocorreu em 2007 após a proposta ter sido submetida ao Programa Nacional de Reorientação da Formação Profissional em Saúde (Pró-Saúde) e resultou na divisão do currículo em eixos, atingindo a carga horária de 8.957 horas.

\section{ABSTRACT}

Introduction: The recent inclusion policies to democratize the access to higher education have urged the development of researches that assess the socioeconomic profile of students, even more in courses of high competition and prestige, such as medicine. Method: In this sense, a cross-sectional study was carried out with the application of tri-analytic questionnaires in a sample of 381 students, with the objective of describing the socioeconomic profile of medical undergraduate students from Universidade Federal da Bahia (UFBA), as well as learning about aspects related to their academic life and future professional performance expectations To analyze the data, SPSS Inc. Software 18 was used in 20 variables that addressed the socioeconomic profile, aspects related to the students' academic life, and career expectations. Results: The study results show that the medical student profile consists of 50.7\% women, 52.8\% of brown ethnicity, mean age of 23 years, 50.4\% from Salvador and its metropolitan region, 38.8\% of the students have a high family income, 55.6\% of them studied me high school years in private schools and for 58\% of the students, college admission did not occur stated that they intend to do a specialization, highlighting the gap in the generalist career, demanded by the Brazilian health system. Conclusion: The results show that even with the implementation of affirmative action policies, the medical course continues to be predominantly an elite course and institutions tend to prioritize teaching spaces, to the detriment of other pillars of the university. 


\section{METODOLOGIA}

Esta pesquisa tem caráter transversal que, segundo Hulley, Cummings, Browner, Grady e Newman ${ }^{14}$, caracteriza-se por ter a coleta de dados realizada em apenas um momento. Esse tipo de delineamento é útil para a descrição de variáveis e dos seus padrões de distribuição.

\section{População e amostra}

O estudo foi realizado com estudantes de graduação que estivessem entre o primeiro e oitavo semestres, já que esse é o período anterior ao internato, e, portanto, essa é uma população de mais fácil acesso. Além disso, desejava-se investigar as expectativas futuras de atuação no mercado de trabalho dos estudantes antes de uma aproximação com a prática profissional, característica do internato. Dessa forma, a população a ser analisada é composta por 713 estudantes, que possuíam matrícula ativa em 2018.1. Foi realizado um cálculo amostral de índice de confiança de $95 \%$. Como o número de questionários respondidos foi superior ao amostral, utilizou-se o total de $\mathrm{n}=381$ estudantes.

\section{Estruturação do instrumento e coleta de dados}

Optou-se pela utilização do instrumento impresso, por ter uma aplicação fácil e simultânea. Dessa forma, foi construído um questionário socioeconômico com questões baseadas no Exame Nacional de Desempenho de Estudantes (Enade). Além das questões de cunho socioeconômico, incluíram-se outras relativas às experiências da vida acadêmica desses alunos e às expectativas e perspectivas futuras da sua atuação profissional. Ao todo, o questionário continha 19 questões.

Os dados foram obtidos a partir da aplicação desse questionário trianalítico. Como o objetivo consistia em garantir a representação de todos os semestres do curso, optou-se por aplicá-lo em sala, durante a avaliação final das disciplinas do eixo ético-humanístico de todas as turmas, do primeiro ao oitavo semestre. Todos os questionários que não foram respondidos integralmente foram excluídos da amostra.

\section{Análise de dados}

Para análise de dados, utilizamos o software SPSS 18, realizando uma análise descritiva das frequências e suas respectivas porcentagens dentro da população estudada. As variáveis estudadas foram: idade (categorizada de acordo com as faixas etárias utilizadas na IV Pesquisa do Perfil Socioeconômico e Cultural dos Estudantes de Graduação das Instituições Federais de Ensino Superior Brasileiras) ${ }^{15}$, gênero, naturalidade, cor/etnia, ensino médio, escolaridade da mãe, escolaridade do pai, renda, forma de ingresso na universidade, recebimento de bolsa-auxílio, recebimento de bolsa acadêmica, possuir graduação anterior, já ter atuado como profissional, fazer ou ter feito estágio, trabalhar atualmente, ter participado de atividade de extensão, ter participado de grupo de pesquisa, ter cursado disciplinas em outros institutos fora da grade curricular padrão, desejo de fazer especialização logo após formado e qual.

\section{Aspectos éticos}

O projeto foi aceito no Comitê de Ética em Pesquisa da Fameb/Ufba e aprovado em julho de 2018 (Certificado de Apresentação para Apreciação Ética (CAAE) no 87862917.8.0000.5531). Todos os estudantes foram informados sobre os objetivos da pesquisa, e aqueles que concordaram em participar da pesquisa assinaram o Termo de Consentimento Livre e Esclarecido em duas vias.

\section{RESULTADOS}

Os resultados apontam que, entre os 381 estudantes, 193 (50,7\%) são do sexo feminino (Tabela 1). A média de idade é de 23 anos, com desvio padrão de 4,297 (63,3\% estão no intervalo entre 20 e 24 anos). Com relação à naturalidade, $192(50,4 \%)$ são de Salvador e da região metropolitana e $59(15,5 \%)$ são de outros estados do Brasil. Quanto à cor/ etnia, $52,8 \%$ se autodeclaram pardos; $32 \%$, brancos; $13,6 \%$, negros; $1,3 \%$ se autodeclara indígena; $0,3 \%$, amarelo.

\begin{tabular}{|c|c|c|}
\hline \multicolumn{3}{|c|}{$\begin{array}{c}\text { Tabela } 1 \\
\text { Perfil socioeconômico e características dos discentes } \\
\text { em Medicina da Fameb }(\mathbf{n}=381)\end{array}$} \\
\hline & $\mathbf{n}$ & $\%$ \\
\hline \multicolumn{3}{|l|}{ Idade } \\
\hline Menor de 20 anos & 52 & $13,6 \%$ \\
\hline De 20 a 24 anos & 241 & $63,3 \%$ \\
\hline De 25 a 29 anos & 55 & $14,4 \%$ \\
\hline Igual ou superior a 30 anos & 33 & $8,7 \%$ \\
\hline \multicolumn{3}{|l|}{ Sexo } \\
\hline Masculino & 188 & $49,3 \%$ \\
\hline Feminino & 193 & $50,7 \%$ \\
\hline \multicolumn{3}{|l|}{ Naturalidade } \\
\hline Salvador e região metropolitana & 192 & $50,4 \%$ \\
\hline Interior da Bahia & 130 & $34,1 \%$ \\
\hline Outro estado & 59 & $15,5 \%$ \\
\hline \multicolumn{3}{|l|}{ Cor/etnia } \\
\hline Branco & 122 & $32,0 \%$ \\
\hline Negro & 52 & $13,6 \%$ \\
\hline Indígena & 5 & $1,3 \%$ \\
\hline Pardo & 201 & $52,8 \%$ \\
\hline Amarelo & 1 & $0,3 \%$ \\
\hline \multicolumn{3}{|l|}{ Ensino médio (maior parte) } \\
\hline Escola pública & 169 & $44,4 \%$ \\
\hline Escola particular & 212 & $55,6 \%$ \\
\hline \multicolumn{3}{|l|}{ Escolaridade da mãe } \\
\hline Nenhuma & 2 & $0,5 \%$ \\
\hline Ensino fundamental completo & 37 & $9,7 \%$ \\
\hline Ensino médio completo & 110 & $28,9 \%$ \\
\hline Ensino superior completo & 116 & $30,4 \%$ \\
\hline Pós-graduação & 116 & $30,4 \%$ \\
\hline \multicolumn{3}{|l|}{ Escolaridade do pai } \\
\hline Nenhuma & 4 & $1,0 \%$ \\
\hline Ensino fundamental completo & 60 & $15,7 \%$ \\
\hline Ensino médio completo & 127 & $33,3 \%$ \\
\hline Ensino superior completo & 114 & $29,9 \%$ \\
\hline Pós-graduação & 76 & $19,9 \%$ \\
\hline
\end{tabular}

\begin{tabular}{l|l}
\hline 34 (2) : e056; 2020 & REVISTA BRASILEIRA DE EDUCAÇÃO MÉDICA \\
\hline
\end{tabular} 


\begin{tabular}{|c|c|c|}
\hline \multicolumn{2}{|c|}{ Tabela 1} & \\
\hline VARIÁVEL & $\mathbf{n}$ & $\%$ \\
\hline \multicolumn{3}{|l|}{ Renda } \\
\hline Até 1,5 salário mínimo & 17 & $4,5 \%$ \\
\hline De 1,5 a 3 salários mínimos & 50 & $13,1 \%$ \\
\hline De 3 a 4,5 salários mínimos & 50 & $13,1 \%$ \\
\hline De 4,5 a 6 salários mínimos & 40 & $10,5 \%$ \\
\hline De 6 a 10 salários mínimos & 76 & $19,9 \%$ \\
\hline De 10 a 30 salários mínimos & 119 & $31,2 \%$ \\
\hline Acima de 30 salários mínimos & 29 & $7,6 \%$ \\
\hline \multicolumn{3}{|l|}{$\begin{array}{l}\text { Ingresso na universidade se deu por } \\
\text { política de afirmação? }\end{array}$} \\
\hline Não & 221 & $58,0 \%$ \\
\hline Sim, por critério étnico-racial & 12 & $3,1 \%$ \\
\hline Sim, por critério de renda & 10 & $2,6 \%$ \\
\hline $\begin{array}{l}\text { Sim, por ter estudado em escola pública } \\
\text { ou particular com bolsa de estudos }\end{array}$ & 53 & $13,9 \%$ \\
\hline $\begin{array}{l}\text { Sim, por sistema que combina dois } \\
\text { ou mais dos critérios anteriores }\end{array}$ & 82 & $21,5 \%$ \\
\hline $\begin{array}{l}\text { Sim, por sistema diferente dos } \\
\text { anteriores }\end{array}$ & 3 & $0,8 \%$ \\
\hline
\end{tabular}

Fonte: Elaborada pelos autores

Ainda, 212 (55,6\%) deles estudaram a maior parte do ensino médio em escola particular. Com relação à escolaridade dos pais, vê-se que a maioria das mães tem superior completo $(30,4 \%)$ e pós-graduação $(30,4 \%)$, enquanto a maioria dos pais tem ensino médio completo $(33,3 \%)$, apesar de a quantidade deles com superior completo também ser representativa $(29,9 \%)$.

Verifica-se que há uma concentração de renda alta na graduação em Medicina, já que 38,8\% afirmam que sua família possui renda maior de 10 salários mínimos. A maioria dos estudantes afirma que o seu ingresso na universidade não se deu por nenhuma política de afirmação (58\%). Além disso, 91,1\% não recebem nenhum tipo de bolsa-auxílio da universidade e $82,9 \%$ não recebem nenhum tipo de bolsa acadêmica (Tabela 2).

Com relação a formações anteriores, $73 \%$ afirmam que se trata da primeira graduação. Quanto à atuação no mercado de trabalho, 81,6\% afirmam que nunca atuaram como profissional, $42,3 \%$ declaram que já fizeram ou fazem estágio e 10,2\% mencionam que trabalham atualmente.

No que se refere a experiências na universidade, $47,2 \%$ já fizeram parte de algum projeto de extensão e $31,5 \%$ já participaram de um grupo de pesquisa. Além disso, apenas 15,5\% afirmam que já cursaram disciplinas em outros institutos fora da grade curricular padrão do curso.

Quanto às expectativas com a atuação profissional, 98,4\% declaram que pretendem fazer especialização após a graduação (Tabela 3). As áreas mais desejadas pelos alunos são: clínica médica (11,7\%), cirurgia $(9,3 \%)$, neurologia $(6,4 \%)$, pediatria (4\%) e psiquiatria (4\%). No entanto, a grande maioria dos estudantes, apesar de manifestar o desejo de fazer especialização, ainda não sabe qual (48,5\%).

\begin{tabular}{|c|c|c|}
\hline \multicolumn{3}{|c|}{$\begin{array}{l}\text { Tabela } 2 \\
\text { lacionados à vida acadêmica dos estudantes de } \\
\text { graduação em Medicina }(\mathrm{n}=381)\end{array}$} \\
\hline VARIÁVEL & $\mathrm{n}$ & $\%$ \\
\hline \multicolumn{3}{|l|}{ Recebe algum tipo de bolsa-auxílio? } \\
\hline Nenhum & 347 & $91,1 \%$ \\
\hline Auxílio-moradia & 5 & $1,3 \%$ \\
\hline Auxílio-alimentação & 0 & $0,0 \%$ \\
\hline Auxílio-moradia e alimentação & 1 & $0,3 \%$ \\
\hline Auxílio-permanência & 19 & $5,0 \%$ \\
\hline Outro tipo de auxílio & 9 & $2,4 \%$ \\
\hline \multicolumn{3}{|c|}{ Recebe algum tipo de bolsa acadêmica? } \\
\hline Nenhum & 316 & $82,9 \%$ \\
\hline Bolsa de iniciação científica & 34 & $8,9 \%$ \\
\hline Bolsa de extensão & 4 & $1,0 \%$ \\
\hline Bolsa de monitoria/tutoria & 10 & $2,6 \%$ \\
\hline Bolsa PET & 8 & $2,1 \%$ \\
\hline Outro tipo de bolsa acadêmica & 9 & $2,4 \%$ \\
\hline \multicolumn{3}{|l|}{ Graduação anterior } \\
\hline Não & 278 & $73,0 \%$ \\
\hline Sim & 103 & $27,0 \%$ \\
\hline
\end{tabular}

Já atuou como profissional?

$\begin{array}{lcc}\text { Não } & 311 & 81,6 \% \\ \text { Sim } & 70 & 18,4 \%\end{array}$

Já fez ou faz estágio?

Não $\quad 220 \quad 57,7 \%$

Sim $\quad 161 \quad 42,3 \%$

Trabalha atualmente?

$\begin{array}{lcc}\text { Não } & 342 & 89,8 \% \\ \text { Sim } & 39 & 10,2 \%\end{array}$

Já participou de algum grupo de extensão?

Não $\quad 201 \quad 52,8 \%$

Sim $\quad 180 \quad 47,2 \%$

Já participou de algum grupo de pesquisa?

$\begin{array}{lll}\text { Não } & 261 & 68,5 \% \\ \text { Sim } & 120 & 31,5 \%\end{array}$

Já cursou alguma disciplina fora da grade curricular padrão de medicina?

$\begin{array}{lcc}\text { Não } & 322 & 84,5 \% \\ \text { Sim } & 59 & 15,5 \%\end{array}$

Pretende fazer especialização?

$\begin{array}{lcc}\text { Não } & 6 & 1,6 \% \\ \text { Sim } & 375 & 98,4 \%\end{array}$

Fonte: Elaborada pelos autores. 


\begin{tabular}{|c|c|c|}
\hline \multicolumn{3}{|c|}{$\begin{array}{c}\text { Tabela } 3 \\
\text { Especialidades pretendidas pelos estudantes de Medicina da Fameb que } \\
\text { indicaram o desejo de fazer especialização após a graduação }(\mathrm{n}=375)\end{array}$} \\
\hline Especialidades pretendidas & $\mathbf{n}$ & $\%$ \\
\hline Não sabe & 182 & $48,5 \%$ \\
\hline Clínica médica & 44 & $11,7 \%$ \\
\hline Cirurgia & 35 & $9,3 \%$ \\
\hline Neurologia & 24 & $6,4 \%$ \\
\hline Pediatria & 15 & $4,0 \%$ \\
\hline Psiquiatria & 15 & $4,0 \%$ \\
\hline Medicina de família e comunidade & 10 & $2,7 \%$ \\
\hline Ortopedia e traumatologia & 9 & $2,4 \%$ \\
\hline Ginecologia e obstetrícia & 8 & $2,1 \%$ \\
\hline Anestesiologia & 5 & $1,3 \%$ \\
\hline Geriatria & 4 & $1,1 \%$ \\
\hline Infectologia & 4 & $1,1 \%$ \\
\hline Neurocirurgia & 4 & $1,1 \%$ \\
\hline Dermatologia & 3 & $0,8 \%$ \\
\hline Otorrinolaringologia & 3 & $0,8 \%$ \\
\hline Patologia & 3 & $0,8 \%$ \\
\hline Acupuntura & 1 & $0,3 \%$ \\
\hline Genética médica & 1 & $0,3 \%$ \\
\hline Medicina de emergência & 1 & $0,3 \%$ \\
\hline Medicina esportiva & 1 & $0,3 \%$ \\
\hline Medicina legal & 1 & $0,3 \%$ \\
\hline Oftalmologia & 1 & $0,3 \%$ \\
\hline Radiologia e diagnóstico por imagem & 1 & $0,3 \%$ \\
\hline
\end{tabular}

Fonte: Elaborada pelos autores.

\section{DISCUSSÃO}

Os resultados obtidos neste estudo descrevem o perfil do estudante da graduação em Medicina da Fameb, sistematizando as características específicas desse grupo.

A média de idade dos estudantes da graduação em Medicina da Ufba permanece próxima da média nacional de 24,5 anos em $2014^{15}$ e também de outros estudos ${ }^{16,17}$. No entanto, vemos uma significativa quantidade de alunos com mais de 30 anos, o que pode significar a busca por melhores condições socioeconômicas ou uma mudança de carreira, já que a medicina é uma das profissões mais rentáveis do país ${ }^{18}$.

Com base nos resultados encontrados, também é possível notar a presença feminina expressiva no curso de Medicina. Isso acompanha um movimento já descrito em alguns estudos sobre a feminização da universidade. Especificamente no Brasil, destaca-se o estudo de Scheffer e Cassenote $^{19}$, segundo os quais houve, pela primeira vez, em 2009 mais novos registros de mulheres nos conselhos regionais do que de homens. O estudo mostrou também que a única faixa etária em que o número de médicas é maior do que de médicos é a que engloba profissionais com menos de 29 anos de idade. Isso quer dizer que as universidades de
Medicina estão formando mais mulheres do que homens atualmente, o que corrobora os resultados obtidos neste estudo.

É importante ressaltar que desde 1994 as mulheres já ocupam a maioria das vagas universitárias $(51,03 \%$ no Nordeste), segundo a primeira pesquisa do perfil socioeconômico e cultural dos estudantes de graduação $^{20}$. De acordo com Queiroz ${ }^{21}$, a Fameb nesse ano era composta por $65,6 \%$ de homens, o que demonstra que essa instituição foi uma das que retardaram a mudança de perfil dos universitários, principalmente por ser historicamente uma profissão masculina ${ }^{19}$.

Apesar de a maioria dos estudantes ter nascido em Salvador e na região metropolitana, o número de graduandos que vieram do interior do estado é muito representativo. É importante destacar também a porcentagem relativamente alta de estudantes oriundos de outros estados (15,5\%), cuja mobilidade foi facilitada pelo Enem e pelo Sisu. Antes, para mudarem de estado para cursar a graduação, os estudantes também tinham que se deslocar para prestar o exame vestibular, o que restringia o acesso.

A Lei de Cotas reserva $50 \%$ das vagas para estudantes advindos do ensino público, acompanhando a proporção populacional de autodeclaração étnico/racial do último censo demográfico, produziu mudanças no perfil dos universitários. Soma-se esse fato a proporção étnica da cidade de Salvador de quase $80 \%$ de pretos/pardos. Por isso, a maioria dos estudantes se autodeclara como tal $(66,4 \%$, somados pretos e pardos), diferentemente de outros estudos realizados em universidades federais pós-cotas que constataram que a maioria dos estudantes ainda se autodeclara branco $^{16,17}$.

A Lei de Cotas também explica o percentual de estudantes que estudaram a maior parte do ensino médio em escolas públicas $(44,4 \%)$. Nota-se que essa taxa é menor do que a estabelecida pela Lei de Cotas (50\%), o que pode indicar a possibilidade de evasão desses estudantes. No entanto, esse resultado é expressivo se comparado ao estudo de Fiorotti, Rossoni e Miranda ${ }^{22}$, realizado no Espírito Santo antes da Lei de Cotas, em que apenas $18,8 \%$ dos estudantes advinham da escola pública. Esse resultado também é coerente com o dado deste estudo de que $58 \%$ dos estudantes afirmam ter entrado na universidade sem utilizar nenhum tipo de política educacional afirmativa.

No entanto, a discussão das cotas relacionando-a com a renda familiar do estudante traz uma contradição, já que, mesmo com elas, $58,7 \%$ dos alunos possuem renda maior que seis salários mínimos. Vale ressaltar que, no Nordeste, apenas 15,67\% dos estudantes universitários (considerando todos os cursos) afirmam ter renda familiar acima de seis salários mínimos ${ }^{15}$. Isso permite inferir que a graduação em Medicina permanece como um curso atrelado às classes médias e altas do país.

Um estudo realizado na Universidade Federal do Espírito Santo (Ufes) no curso de Medicina ${ }^{22}$, no período pré-cotas, expõe que 57,2\% dos graduandos tinham uma renda familiar maior que cinco mil reais um dado muito próximo ao encontrado nesta pesquisa, o que demonstra que a Lei de Cotas ainda não contribuiu expressivamente para a inclusão de estudantes economicamente vulneráveis na graduação em Medicina. Ristoff $^{11}$ evidencia que os estudantes de Medicina apresentam as maiores rendas familiares em comparação com os demais cursos de graduação. Ao analisar o perfil socioeconômico dos estudantes universitários brasileiros entre 1991 e 2012 com os dados do Enade, o pesquisador afirma que os estudantes desse curso são seis vezes mais abastados que a população brasileira em geral.

\begin{tabular}{l|l}
\hline REVISTA BRASILEIRA DE EDUCAÇÃO MÉDICA \\
\hline 44 (2) : e056; 2020
\end{tabular} 
Com relação à escolaridade dos pais, percebe-se que a maioria $(60,8 \%$ das mães e 49,8\% dos pais) dos estudantes informou que eles concluíram o ensino superior, evidenciando que essa média é superior à média do Nordeste, segundo estudo com todas as graduações das instituições federais $^{15}$. Mas, se comparada com outras graduações em Medicina, essa média obedece à tendência para o curso. Além disso, também está de acordo com a tendência nacional em que as mães possuem maior escolaridade do que os pais.

Vale salientar que há uma forte correlação entre origem social e a situação econômica familiar do estudante: discentes com pais que têm escolaridade superior possuem rendas mais elevadas, e essa população estudantil está matriculada em cursos de alta concorrência. Dados do Enade de 1991-2012 apontam que 43\% dos estudantes de Medicina tinham pais com escolaridade superior, enquanto apenas $5 \%$ dos discentes de pedagogia apresentavam perfil idêntico ${ }^{11}$.

Sobre possuir outra graduação, $73 \%$ dos estudantes informaram não possuir diploma de formação superior e $81,1 \%$ mencionaram que nunca haviam exercido uma profissão; além disso, $89,8 \%$ dos informantes não trabalham atualmente. Esses dados indicam que a maior parte dos discentes de Medicina tem esse curso como primeira graduação e dedica-se integralmente aos "estudos". A dedicação integral à formação universitária é possível, na maioria dos casos, com o suporte financeiro familiar ou por meio de algum auxílio-permanência concedido pela universidade. No caso dos estudantes de Medicina da Fameb, 91,1\% afirmaram não receber nenhum tipo de bolsa-auxílio, e isso nos leva a concluir que ou os estudantes recebem suporte financeiro da família, o que corrobora a renda informada neste estudo, ou que uma parcela desses estudantes está em situação de vulnerabilidade socioeconômica, o que pode comprometer a permanência deles na universidade ${ }^{18}$.

Outro ponto que merece ser destacado é que, no caso da formação médica, que possui alta carga horária curricular e aulas em mais de um turno, os estudantes ficam impossibilitados de encontrar empregos fixos, ou seja, a formação universitária em Medicina é pouco flexível, tornando difícil conciliar estudos com o trabalho ${ }^{23}$. Não foi possível identificar literatura que discuta as experiências universitárias de estudantestrabalhadores no curso de Medicina, no entanto, em outras profissões da saúde, como o caso da enfermagem, a presença de estudantestrabalhadores é uma realidade ${ }^{24}$.

No que tange à inserção em projetos de pesquisa, $68,5 \%$ dos estudantes informaram nunca ter participado de nenhum grupo, evidenciando dessa maneira que o tripé que fundamenta a universidade (ensino, pesquisa e extensão) não se efetiva, e aqui se encontra dissociado. Além disso, essa postura de não inserir os discentes de Medicina na pesquisa científica vai na contramão do movimento que tem sido incentivado para a formação profissional em saúde: a prática médica baseada em evidências ${ }^{25}$.

A respeito do desenvolvimento de atividades de extensão, 52,8\% dos informantes declararam nunca ter participado desses tipos de projetos. Ayres $^{26}$ chama a atenção para a importância de práticas de extensão na formação médica, pois elas possibilitam aos estudantes, quando em contato com comunidades e famílias, estabelecer interações diferentes das desenvolvidas nos serviços de saúde, constituindo assim diversificado cenário de ensino-aprendizagem.

Uma possibilidade explicativa atrelada a essa baixa adesão nas atividades de pesquisa e extensão é a sobrecarga que os estudantes de
Medicina enfrentam ao longo do curso: alta carga horária, imensa quantidade de informações, grande volume de estudos e cobranças para o desenvolvimento de habilidades técnicas ${ }^{23}$. Em pesquisa realizada na Ufes, $79 \%$ dos estudantes de medicina declararam que se sentiam sobrecarregados com o curso de Medicina ${ }^{22}$.

Dessa maneira, os estudantes de Medicina não encontram oportunidades para cursar disciplinas fora da grade curricular padrão: 84,5\% dos discentes da Fameb informaram que nunca haviam participado de componentes curriculares diferentes daqueles a que são obrigados ou com os quais estão habituados.

Outro aspecto encontrado na literatura que tem discutido a sobrecarga dos discentes de medicina é o fenômeno do "currículo paralelo", atividades extracurriculares que não são integralizadas na matriz curricular, nas quais os estudantes têm se envolvido para aperfeiçoar a prática clínica e adquirir um bom currículo para assim facilitar a entrada nas residências médicas ${ }^{27}$. Duas pesquisas, uma realizada na Universidade Federal de Minas Gerais (UFMG) ${ }^{27}$ e outra na Universidade Federal de Alagoas (Ufal) ${ }^{28}$, encontraram evidências de que a grande maioria dos estudantes realizou alguma atividade extracurricular com os fins já mencionados: $82,5 \%$ estudantes da UFMG e $98,4 \%$ da Ufal estão desenvolvendo currículo paralelo, e 55,77\% dessas atividades consomem mais de 12 horas semanais.

Quanto à pretensão de fazer especialização, 98,4\% dos estudantes - um resultado expressivo - informaram que pretendem ingressar num programa de residência após a conclusão do bacharelado. Em pesquisa semelhante realizada na Universidade Federal de Goiás (UFG), 96,3\% dos discentes declararam que pretendem realizar residência, dos quais $83,7 \%$ desejam ser especialistas ${ }^{29}$.

Apesar de desejarem fazer uma residência, parte considerável dos respondentes desta pesquisa, $48,5 \%$, declarou não saber qual especialidade pretende seguir; em contrapartida, 51,5\% dos estudantes mencionaram 22 especialidades diferentes. Tais evidências contribuem para inferir que as expectativas de carreira dos estudantes de Medicina não estão alinhadas com as necessidades do SUS, pois a superespecialização na área médica se distancia do trabalho interprofissional e colaborativo, assim como das competências necessárias para a resolução dos problemas de saúde que surgem na atenção primária à saúde (APS) ${ }^{30}$.

Ceccim et al. ${ }^{31}$ oferecem uma chave analítica interessante para pensar as expectativas sobre a atuação profissional. De acordo com esses autores, há no processo formativo em saúde um imaginário liberal e privatista quanto à atuação profissional. Os estudantes de Medicina, Odontologia e Psicologia que participaram desse estudo empírico veem no serviço público uma segurança financeira, mas $80 \%$ apontam que o sucesso profissional está relacionado com a atuação em clínicas privadas. No entanto, sabe-se que a assistência médica nessas instituições está baseada no atendimento individual e curativo, realizando apenas assistência e recuperação, sem considerar a promoção da saúde e a prevenção de doenças, como é preconizado no SUS.

O processo de trabalho centrado na multiplicidade de especialidades tem produzido um esvaziamento na função dos médicos generalistas e das especialidades-raízes (pediatria, cirurgia geral, clínica médica, ginecoobstetrícia e saúde pública). Além disso, a incorporação dessas "novas" especialidades aos sistemas de saúde é extremamente onerosa ${ }^{32}$. 


\section{CONCLUSÃO}

O perfil do estudante de Medicina da Fameb é predominantemente feminino, pardo, com média de 23 anos, oriundo de Salvador e da região metropolitana. Além disso, possui alta renda familiar, estudou a maior parte do ensino médio em escola particular e a entrada no curso não se deu por política de afirmação. A grande maioria não recebe nenhum tipo de auxílio da universidade ou bolsa acadêmica e, além disso, não participa de grupo de extensão, de pesquisa ou cursa disciplinas que não esteja dentro da sua grade curricular padrão. Medicina, para a maioria, é a primeira graduação, e, além disso, o estudante nunca trabalhou, não fez ou faz estágio e não trabalha durante a graduação. Quase a totalidade dos estudantes pretende fazer especialização depois de formado.

A discussão sobre o currículo médico evidencia um tensionamento entre as políticas existentes e os interesses dessa corporação profissional. Dessa forma, o estudo mostra que, mesmo com a implementação das políticas de ação afirmativa que passaram a garantir a entrada populações historicamente excluídas na universidade, o curso de Medicina continua sendo predominantemente um curso de elite. Além disso, infere-se que a faculdade de Medicina não tem promovido espaços e oportunidades para que os estudantes participem das atividades de extensão e pesquisa, priorizando o ensino em detrimentos dos outros pilares da universidade. Os resultados do estudo também demonstram e confirmam a excessiva busca pela especialização e a consequente lacuna na formação de médicos que sigam a carreira generalista.

\section{AGRADECIMENTO}

Agradecemos aos bolsistas de Iniciação Científica Jarbas Carneiro Mota e Gabriela Flor Martins a colaboração na aplicação dos questionários na Faculdade de Medicina da Ufba.

\section{REFERENNCIAS}

1. Ciuffo RS, Ribeiro VMB. Sistema Único de Saúde e a formação dos médicos: um diálogo possível? Interface (Botucatu) 2008;12(24):125-40.

2. Carvalho MN, Gil CRR, Costa EMOD, Sakai MH, Leite SN. Necessidade e dinâmica da força de trabalho na Atenção Básica de Saúde no Brasil. Ciênc. Saúde Col. 2018;23(1):295-302. doi: 10.1590/1413-81232018231.08702015.

3. Paim J, Travassos C, Almeida C, Bahia L, Macinko J. O sistema de saúde brasileiro: história, avanços e desafios. Lancet 2011;377(9779):1778-97.

4. Veras RM, Feitosa CCM. Reflexões em torno das Diretrizes Curriculares Nacionais do curso de graduação em Medicina a partir da Política Nacional de Promoção da Saúde. Interface (Botucatu) 2019;23(sup 1):e170743. doi: 10.1590/interface.170743.

5. Brasil. Ministério da Educação. Conselho Nacional de Educação, Câmara de Educação Superior. Resolução no 4 , de 7 de novembro de 2001. Institui Diretrizes Curriculares Nacionais do Curso de Graduação em Medicina. Brasília; 2001.

6. Brasil. Ministério da Educação. Conselho Nacional de Educação, Câmara de Educação Superior. Resolução no 3, de 20 de junho de 2014. Institui Diretrizes Curriculares Nacionais do Curso de Graduação em Medicina e dá outras providências. Brasília; 2014.

7. Gonzaga HN, Kormann SO. A carga horária excessiva do curso de graduação em medicina e sua repercussão na saúde mental do estudante. Cadernos Brasileiros de Saúde Mental 2014;6(13):156.

8. Formigli VL, Barbosa HS, Lima MAG, Araújo IB, Fagundes NC, Macedo, RSA. Projeto Político-Pedagógico do curso de graduação em Medicina da FMB/UFBA. Gazeta Médica da Bahia 2010;79(1):3-47.

9. Universidade Federal da Bahia. Resolução no 03/2013. Dispõe sobre a adoção do Exame Nacional do Ensino Médio- ENEM e sobre a adesão ao Sistema de Seleção Unificada -SISU/MEC, para ingresso nos cursos de graduação da UFBA. 19 de junho de 2013.

10. Brasil. Lei $\mathrm{n}^{\circ} 12.711$, de 29 de agosto de 2012. Dispõe sobre o ingresso nas universidades federais e nas instituições federais de ensino técnico de nível médio e dá outras providências. Brasília, DF [acesso em 10 mar 2013]. Disponível em: http://www. planalto.gov.br/ccivil_03/_ ato2011-2014/2012/lei/112711.htm.

11. Ristoff D. O novo perfil do campus brasileiro: uma análise do perfil socioeconômico do estudante de graduação. Avaliação: Revista da Avaliação da Educação Superior (Campinas) 2014;19(3):723-47. doi: 10.1590/S1414-40772014000300010.

12. Lamounier JA, Torga ALGF, Leite GLF. Características e conceitos dos formandos do curso de Medicina da UFMG. Pediatria (São Paulo) 2002;24(3-4):85-92.

13. Almeida Filho N. Bacharelado interdisciplinar em Saúde: revolução na educação superior no campo da saúde? In: Teixeira FT, Coelho MTD, organizadores. Uma experiência inovadora no ensino superior: bacharelado interdisciplinar em Saúde. Salvador: Edufba; 2014. p. 11-22.

14. Hulley SB, Cummings SR, Browner WS, Grady DG, Newman TB. Delineando a pesquisa clínica: uma abordagem epidemiológica. $3 \mathrm{a}$ ed. Porto Alegre: Artmed; 2008.

15. Fórum Nacional de Pró-Reitores de Assuntos Comunitários e Estudantis, Associação Nacional dos Dirigentes das Instituições Federais de Ensino Superior. IV Pesquisa do Perfil Socioeconômico e Cultural dos Estudantes de Graduação das Instituições Federais de Ensino Superior Brasileiras. Brasília: Fonaprace, Andifes; 2016.

16. Bampi LNS, Baraldi S, Guilhem D, Araújo MP, Campos ACO. Qualidade de vida de estudantes de Medicina da Universidade de Brasília. Rev. bras. educ. med. 2013;37(2):217-25.

17. Cardoso Filho FAB, Magalhães JF, Silva KML, Pereira ISSD. Perfil do estudante de Medicina da Universidade do Estado do Rio Grande do Norte (UERN), 2013. Rev. bras. educ. med. 2015;39(1):32-40.

18. Silva SA. Travessias improváveis - permanência de estudantes das camadas populares no curso de Medicina da Universidade Federal da Bahia [dissertação]. Salvador: Universidade Federal da Bahia; 2018.

19. Scheffer MC, Cassenote AJF. A feminização da medicina no Brasil. Rev. bioét. (Impr.) 2013;21(2):268-77.

20. Fórum Nacional de Pró-Reitores de Assuntos Comunitários e Estudantis, Associação Nacional dos Dirigentes das Instituições Federais de Ensino Superior. I Pesquisa Nacional de Perfil Socioeconômico e Cultural dos Graduandos da IFES. Brasília: Fonaprace, Andifes; 1994.

21. Queiroz DMQ. Mulheres no ensino superior no Brasil. 23a Reunião Anual Anped; set 2000 [acesso em 25 jun 2019]. Disponível em: http://23reuniao.anped.org.br/textos/0301t.PDF.

22. Fiorotti KP, Rossoni RR, Miranda AE. Profile of medical students at the Federal University in Espírito Santo, Brazil, 2007. Rev. bras. educ. med. 2010;34(3):355-62. 
23. Dini PS, Batista NA. Graduação e prática médica: expectativas e concepções de estudantes de Medicina do $1^{\circ}$ ao $6^{\circ}$ ano. Rev. bras. educ. med. 2004;28(3):198-203.

24. Costa MLAS, Merighi MAB, Jesus MCP. Ser enfermeiro tendo sido estudante-trabalhador de enfermagem: um enfoque da fenomenologia social. Acta paul. enferm. 2008;21(1):17-23.

25. Wannmacher L, Fuchs FD. Conduta terapêutica embasada em evidências. Rev. Ass. Med. Bras. 2000;46(3):237-41.

26. Ayres JRCM. Extensão universitária: aprender fazendo, fazer aprendendo. Rev. med. (São Paulo) 2015;94(2):75-80.

27. Tavares AP, Ferreira RA, França RB, Fonseca Junior CA, Lopes GC, Dantas NGT, et al. O “currículo paralelo" dos estudantes de Medicina da Universidade Federal de Minas Gerais. Rev. bras. educ. med. 2007;31(3):254-65.

28. Tavares CHF, Maia JA, Muniz MCH, Malta MV, Magalhães BRC, Thomaz ACP. O currículo paralelo dos estudantes da terceira série do curso Médico da Universidade Federal de Alagoas. Rev. bras. educ. med. 2007;31(3):245-53.

29. Filisbino MA, Moraes VA. A graduação médica e a prática profissional na perspectiva de discentes. Rev. bras. educ. med. 2013;37(4):540-8.

30. Peduzzi M, Agreli HF. Teamwork and collaborative practice in Primary Health Care. Interface (Botucatu) 2018;22(supl 2):1525-34.

31. Ceccim RB, Armani TB, Oliveira DLLC, Bilibio LF, Moraes M, Santos ND. Imaginários da formação em saúde no Brasil e os horizontes da regulação em saúde suplementar. Ciênc. Saúde Colet. 2008;13(5):156778. doi: 10.1590/S1413-81232008000500021.
32. Campos GWS, Chakour M, Santos RC. Análise crítica sobre especialidades médicas e estratégias para integrá-las ao Sistema Único de Saúde (SUS). Cad. saúde pública 1997;13(1):141-4.

\section{CONTRIBUIÇÃO DOS AUTORES}

Renata Meira Veras participou das seguintes etapas do desenvolvimento deste manuscrito: desenho do estudo, revisão da literatura, elaboração e adaptação do questionário e análise e revisão crítica do texto. Clara Couto Fernandez participou da revisão da literatura, elaboração e adaptação do questionário, coleta e análise de dados e elaboração da versão inicial e final do artigo. Caio Cezar Moura Feitosa participou da revisão da literatura, elaboração e adaptação do questionário, análise de dados e elaboração da versão inicial e final do artigo. Sheyla Fernandes participou da análise de dados e revisão crítica do texto.

\section{CONFLITO DE INTERESSES}

Os autores declaram não haver conflito de interesses neste estudo.

\section{ENDEREÇO PARA CORRESPONDÊNCIA}

Renata Meira Veras. Instituto de Humanidades, Artes e Ciências Professor Milton Santos, Rua Barão de Jeremoabo, s/n, PAF V, Ondina, Salvador, BA, Brasil. CEP: 40170-115.

E-mail: renata.veras@ufba.br 